\title{
Heparin-induced thrombocytopenia presenting as unilateral lower limb paralysis following lumbar spine surgery: case report
}

\author{
Brandon W. Smith, MD, Jacob R. Joseph, MD, and Paul Park, MD \\ Department of Neurosurgery, University of Michigan, Ann Arbor, Michigan
}

Heparin-induced thrombocytopenia (HIT) is a state of thrombocytopenia with a paradoxically elevated thrombotic potential after exposure to heparin. Severe cases can present with multiorgan involvement with direct and secondary effects. Although HIT has been reported following other surgeries, to the authors' knowledge there has not been a report of HIT after spinal surgery. The present case details the course of a patient who underwent elective lumbar surgery followed by delayed presentation of shortness of breath due to multiple pulmonary embolisms and right lower-extremity paralysis due to extensive iliofemoral clot burden with acute compartment syndrome. The patient was treated with intravenous argatroban for extensive thrombosis and also required open thrombectomy and fasciotomies for treatment of compartment syndrome. Although the patient eventually experienced motor recovery, residual sensory deficits persisted at last follow-up. In this report, the pathophysiology, clinical presentation, and treatment of HIT are reviewed.

https://thejns.org/doi/abs/10.3171/2016.10.SPINE16996

KEY WORDS heparin-induced thrombocytopenia; spinal surgery; lumbar spine; complication

$\mathrm{H}$ EPARIN-INDUCED thrombocytopenia (HIT) with or without thrombosis is a rare, but well-described clinical condition that can develop after exposure to heparin. ${ }^{6}$ The presence of HIT is characterized by development of thrombocytopenia after exposure to heparin. In some cases this causes a paradoxically greater tendency for clot formation, ${ }^{6}$ resulting in thrombotic events. In the most extreme cases, the clot burden can be so extensive that patients present with multiorgan involvement and injury necessitating aggressive medical and invasive treatments.

Here, we present a case of HIT with extensive thrombosis in a patient who had undergone a recent spine operation with exposure to postoperative unfractionated heparin prophylaxis. This patient went on to develop compartment syndrome that caused acute lower-extremity paralysis, renal failure, deep venous thromboses (DVTs), and pulmonary embolisms (PEs) causing respiratory failure. The purpose for presenting this case is to increase the awareness that HIT can develop in spine patients after prophy- lactic dosing of unfractionated heparin, and that this can present as acute weakness, among other symptoms. Increased awareness can aid in earlier recognition, diagnosis, and initiation of treatment for this potentially dangerous condition.

\section{Case Report}

A 59-year-old woman presented to our neurosurgery clinic with a 2-year history of progressive chronic back and right leg pain. Conservative measures had failed, including oral pain medications, physical therapy, and steroid injections. Physical examination demonstrated full strength and sensation with intact reflexes. MRI demonstrated a rightsided L4 -5 synovial cyst resulting in severe spinal stenosis with a Grade 1 spondylolisthesis. Surgical plans included laminectomy, resection of the cyst, and interbody fusion. Preoperative workup revealed a platelet count of $155 / \mu 1$.

The patient underwent the planned procedure without a dose of intraoperative heparin, and her arterial line was un-

ABBREVIATIONS DVT = deep venous thrombosis; $\mathrm{HIT}=$ heparin-induced thrombocytopenia; $\mathrm{PE}=$ pulmonary embolism; $\mathrm{POD}=$ postoperative day .

SUBMITTED August 23, 2016. ACCEPTED October 14, 2016.

INCLUDE WHEN CITING Published online February 3, 2017; DOI: 10.3171/2016.10.SPINE16996. 


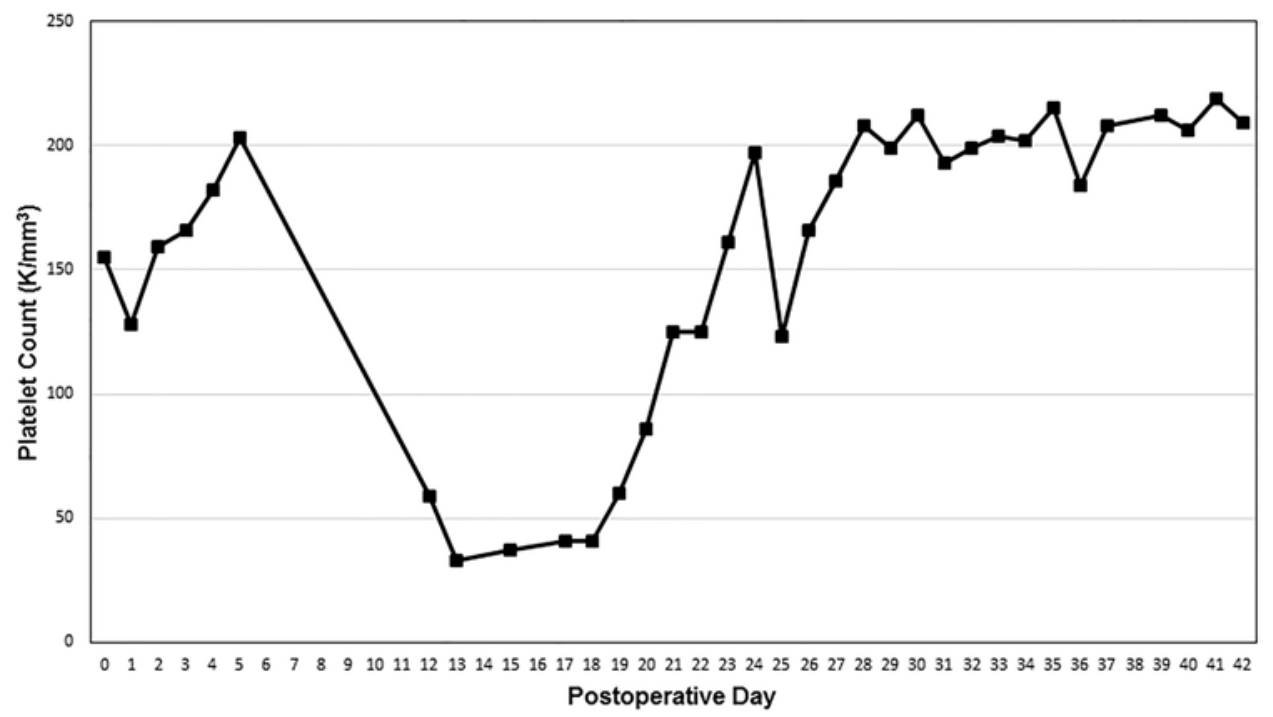

FIG. 1. Platelet count trends starting at postoperative Day 0.

heparinized. The procedure was well tolerated, and postoperative imaging demonstrated adequate placement of hardware. Postoperatively the patient's strength remained intact, and she was started on a regimen of prophylactic heparin (5000 U subcutaneously 3 times daily) at 10:00 PM on postoperative day (POD) 1. Prior to initiation of the heparin prophylaxis on POD 1, the patient's platelet count was 128/ $\mu 1$, and on the day of discharge (POD 7), her platelet count was 203/ul (Fig. 1). She continued receiving heparin prophylaxis for the duration of her initial hospitalization. Her postoperative course was complicated by an ileus, which resolved with bowel rest. The patient was discharged on POD 7 with a stable intact neurological examination.

On POD 14 the patient presented to the emergency department, where she was found to be in severe respiratory distress with acute lower-extremity weakness (4/5 left dorsiflexion and hip flexion, and 0/5 in her right lower extremity throughout). She had intact sensation at the level of her navel but denied sensation throughout her legs bilaterally. Her legs were cool to the touch and mottled in appearance. She had palpable pulses in the upper extremities and groin, but distal to her groin she did not have palpable pulses or pulses evident on Doppler ultrasonography. Due to respiratory distress, the patient was intubated, and workup revealed multiple PEs, a renal infarct, and DVTs in both her iliac and femoral veins bilaterally. Her platelet count was $59 / \mu \mathrm{l}$ and her HIT immunoassay was positive. She was started on intravenous argatroban. Based on her lower-extremity findings, the patient was diagnosed with acute compartment syndrome and was taken to the operating room for bilateral groin cut-downs, bilateral iliofemoral thrombectomies, and right anterior/lateral/posterior fasciotomies. She had a prolonged recovery from her multisystem injury but was eventually discharged on hospitalization Day 31 with 1-2/5 strength in her right lower extremity and full recovery of her left lower-extremity strength. She was discharged on a regimen of long-term warfarin. The patient was last seen for follow-up approximately 7 months after her initial operation and 6.5 months after the HIT presentation. She returned to full strength on physical examination; however, she still had limited sensation and ambulated with a noticeable limp.

\section{Discussion}

HIT is a rare disease that can develop after exposure to heparin. Clinical manifestation includes thrombocytopenia and a paradoxically elevated thrombotic state ${ }^{6}$ that develops 5-10 days after exposure., ${ }^{6,22}$ The mechanism for development of HIT depends on immunoglobulin G immune complexes that activate platelets, monocytes, and endothelium, ultimately increasing the generation of thrombin and creating a prothrombotic state. ${ }^{3,9,23}$

Our patient presented with multiple venous and arterial thrombotic events. Approximately half of the patients with HIT present initially with a thrombosis. Of those patients who are diagnosed with HIT without thrombosis, 52.8\% will go on to develop a thrombotic event within the next 30 days. ${ }^{21}$ DVT and PE are the most common thrombotic complications, with incidences of $48 \%$ and $25 \%$, respectively. ${ }^{21}$ In addition to venous thrombosis, there are reports of limb artery thrombosis, myocardial infarction, and cerebrovascular accident; however, the ratio of venous to arterial thrombosis is 4:1. ${ }^{21}$ Initial concern was for a spinal cord stroke or cerebrovascular accident; however, MRI findings were unrevealing. Compartment syndrome with peripheral nerve compression secondary to extensive DVT burden was the diagnosis for the patient's weakness.

HIT develops in approximately 1 in 5000 hospitalized patients. ${ }^{6}$ Within the surgical population, most reported cases result from cardiovascular surgery and nonspine orthopedic surgery. ${ }^{22}$ There are reports in the literature of HIT in neurovascular ${ }^{8}$ and neuro-oncology ${ }^{10,16}$ patients; however, there are no reports of HIT in degenerative spine surgery patients. The risk of HIT appears to be slightly higher in women ${ }^{7}$ and appears to be up to 10 times higher in patients exposed to unfractionated heparin than those exposed to low-molecular-weight heparin; ${ }^{15}$ both risk factors were present in our patient. The evidence demonstrat- 
TABLE 1. The 4-T's scale scoring in the presented case

\begin{tabular}{llc}
\hline \multicolumn{1}{c}{ Parameter } & \multicolumn{1}{c}{ Determinant } & Score* $^{*}$ \\
\hline Thrombocytopenia & $\begin{array}{c}\text { Platelet count fall }>50 \% \text { \& platelet nadir } \\
\geq 20\end{array}$ & 2 \\
\hline $\begin{array}{c}\text { Timing of platelet } \\
\text { count decrease }\end{array}$ & $\begin{array}{c}\text { Consistent w/ Day } 5-10 \text { decrease, but } \\
\text { unclear due to missing platelet counts }\end{array}$ & 1 \\
\hline Thrombosis & New thrombosis & 2 \\
\hline Other causes & None apparent & 2 \\
\hline
\end{tabular}

* A total of 7 is a high score.

ing an increased risk of HIT with unfractionated heparin in comparison with low-molecular-weight heparin has been growing in the cardiac surgery literature, neurology literature, and trauma literature.,13,15,18 These data have led to some institutions transitioning to low-molecular-weight heparin for DVT prophylaxis.

The diagnosis of HIT becomes likely with a $50 \%$ or greater decrease in platelets beginning 5-10 days after initiating heparin therapy and with the presence of HIT antibodies. ${ }^{6,22}$ Our patient presented outside this window; however, we did not have platelet counts from Days 8-13 (Fig. 1). Thrombocytopenia can occur from multiple etiologies in medical and surgical patients. ${ }^{4}$ However, a predictive scale has been developed to evaluate the likelihood of HIT based on the clinical syndrome. ${ }^{12}$ The "4-T's" scale consists of thrombocytopenia parameters, timing of presentation, the presence of thrombotic events, and the likelihood of alternative causes. ${ }^{12}$ A high score on the 4-T's scale has a high positive predictive value, and a low score has a high negative predictive value; however, intermediate scoring is less clinically useful. ${ }^{12}$ Our patient had a high score due to positive factors in all 4 areas (Table 1). Clinical diagnosis alone is often not enough to be definitive. Immunoassays can be used in combination with the 4-T's scale to verify a diagnosis. The various assays used have a high negative predictive value, but their false-positive rate is high, making their isolated use troublesome. ${ }^{6}$

Despite laboratory evidence of thrombocytopenia, the risk of hemorrhage in HIT is very low. As such, platelet transfusion is not indicated and can instead increase thrombotic events. ${ }^{5}$ The complications that our patient experienced were directly related or secondary to thrombotic events; she did not have any bleeding events.

There are currently 2 approved treatments for HITargatroban ${ }^{11,20}$ and danaparoid. ${ }^{2}$ Argatroban is a direct thrombin inhibitor, and danaparoid is an antithrombindependent factor Xa inhibitor. Both of these medications have proven to be effective at decreasing thrombosis..1 Our patient was treated with argatroban until her platelet count recovered and she could be transitioned to long-term warfarin anticoagulation. Danaparoid is also available in a subcutaneous formulation, which could have potential for outpatient therapy if indicated.

As part of her HIT presentation, the patient had asymmetrical weakness due to compartment syndrome that developed secondary to the lower-extremity thrombosis. Weakness in compartment syndrome can be multimodal, including muscle ischemia, nerve entrapment, and guarding from severe pain. ${ }^{17}$ It is likely that this patient's initial presentation was a mixture of these various etiologies, as she had hip flexor weakness in addition to paralysis distal to the knee. She underwent open thrombectomies bilaterally and fasciotomies of the right lower extremity. The patient's strength improved throughout her hospitalization, and at her 6-month follow-up she had no perceptible weakness on physical examination. This case illustrates that compartment syndrome is a surgical emergency, ${ }^{14,19}$ and that early recognition and treatment is important for recovery. ${ }^{14}$

\section{Conclusions}

We have presented a case of HIT with multiorgan involvement in a patient who underwent elective lumbar spine surgery with postoperative prophylactic exposure to unfractionated heparin. Overall, HIT is a rare clinical manifestation that has been demonstrated in both neurooncology and neurovascular patients; however, this is the first report of HIT in a spine surgery patient.

\section{References}

1. Bucci C, Geerts WH, Sinclair A, Fremes SE: Comparison of the effectiveness and safety of low-molecular weight heparin versus unfractionated heparin anticoagulation after heart valve surgery. Am J Cardiol 107:591-594, 2011

2. Chong BH, Gallus AS, Cade JF, Magnani H, Manoharan A, Oldmeadow M, et al: Prospective randomised open-label comparison of danaparoid with dextran 70 in the treatment of heparin-induced thrombocytopaenia with thrombosis: a clinical outcome study. Thromb Haemost 86:1170-1175, 2001

3. Cines DB, Tomaski A, Tannenbaum S: Immune endothelialcell injury in heparin-associated thrombocytopenia. N Engl J Med 316:581-589, 1987

4. Erkurt MA, Kaya E, Berber I, Koroglu M, Kuku I: Thrombocytopenia in adults: review article. J Hematol 1:44-53, 2012

5. Goel R, Ness PM, Takemoto CM, Krishnamurti L, King KE, Tobian AA: Platelet transfusions in platelet consumptive disorders are associated with arterial thrombosis and in-hospital mortality. Blood 125:1470-1476, 2015

6. Greinacher A: Clinical practice. Heparin-induced thrombocytopenia. N Engl J Med 373:252-261, 2015

7. Greinacher A, Warkentin TE: Risk of heparin-induced thrombocytopenia in patients receiving thromboprophylaxis. Expert Rev Hematol 1:75-85, 2008

8. Gupta V, Tanvir R, Garg A, Gaikwad SB, Mishra NK: Heparin-induced thrombocytopenia in a case of endovascular aneurysm coiling. AJNR Am J Neuroradiol 28:155-158, 2007

9. Kelton JG, Sheridan D, Santos A, Smith J, Steeves K, Smith C, et al: Heparin-induced thrombocytopenia: laboratory studies. Blood 72:925-930, 1988

10. Kruljac I, Cerina V, Pećina HI, Pažanin L, Matić T, Božikov $\mathrm{V}$, et al: Pituitary metastasis presenting as ischemic pituitary apoplexy following heparin-induced thrombocytopenia. Endocr Pathol 23:264-267, 2012

11. Lewis BE, Wallis DE, Hursting MJ, Levine RL, Leya F: Effects of argatroban therapy, demographic variables, and platelet count on thrombotic risks in heparin-induced thrombocytopenia. Chest 129:1407-1416, 2006

12. Lo GK, Juhl D, Warkentin TE, Sigouin CS, Eichler P, Greinacher A: Evaluation of pretest clinical score (4 T's) for the diagnosis of heparin-induced thrombocytopenia in two clinical settings. J Thromb Haemost 4:759-765, 2006

13. Lubenow N, Hinz P, Thomaschewski S, Lietz T, Vogler M, Ladwig A, et al: The severity of trauma determines 
the immune response to PF4/heparin and the frequency of heparin-induced thrombocytopenia. Blood 115:1797-1803, 2010

14. Mabvuure NT, Malahias M, Hindocha S, Khan W, Juma A: Acute compartment syndrome of the limbs: current concepts and management. Open Orthop J 6:535-543, 2012

15. Martel N, Lee J, Wells PS: Risk for heparin-induced thrombocytopenia with unfractionated and low-molecular-weight heparin thromboprophylaxis: a meta-analysis. Blood 106:2710-2715, 2005

16. Nitta N, Shitara S, Nozaki K: Heparin-induced thrombocytopenia in a glioblastoma multiforme patient with inferior vena cava filter placement for deep venous thrombosis. Neurol Med Chir (Tokyo) 51:445-448, 2011

17. Olson SA, Glasgow RR: Acute compartment syndrome in lower extremity musculoskeletal trauma. J Am Acad Orthop Surg 13:436-444, 2005

18. Pohl C, Kredteck A, Bastians B, Hanfland P, Klockgether $T$, Harbrecht U: Heparin-induced thrombocytopenia in neurologic patients treated with low-molecular-weight heparin. Neurology 64:1285-1287, 2005

19. Via AG, Oliva F, Spoliti M, Maffulli N: Acute compartment syndrome. Muscles Ligaments Tendons J 5:18-22, 2015

20. Warkentin TE, Greinacher A, Koster A, Lincoff AM: Treatment and prevention of heparin-induced thrombocytopenia: American College of Chest Physicians Evidence-Based Clinical Practice Guidelines (8th Edition). Chest 133 (6 Suppl):340S-380S, 2008
21. Warkentin TE, Kelton JG: A 14-year study of heparin-induced thrombocytopenia. Am J Med 101:502-507, 1996

22. Warkentin TE, Kelton JG: Temporal aspects of heparininduced thrombocytopenia. N Engl J Med 344:1286-1292, 2001

23. Ziporen L, Li ZQ, Park KS, Sabnekar P, Liu WY, Arepally $\mathrm{G}$, et al: Defining an antigenic epitope on platelet factor 4 associated with heparin-induced thrombocytopenia. Blood 92:3250-3259, 1998

\section{Disclosures}

Dr. Park reports that he is a consultant for Globus, Medtronic, Biomet, and NuVasive, and he receives royalties from Globus.

\section{Author Contributions}

Conception and design: Park. Acquisition of data: all authors. Analysis and interpretation of data: all authors. Drafting the article: Smith, Joseph. Critically revising the article: all authors. Reviewed submitted version of manuscript: all authors. Approved the final version of the manuscript on behalf of all authors: Park. Study supervision: Park.

\section{Correspondence}

Paul Park, Department of Neurosurgery, University of Michigan, 1500 E Medical Center Dr., Rm. 3553 TC, Ann Arbor, MI 481095338. email: ppark@med.umich.edu. 